\title{
Targeted Mutagenesis of Beta-Lactoglobulin Gene in Caprine Fetal Fibroblasts by Context-Dependent Assembly Zinc-Finger Nucleases
}

\author{
Yuguo Yuan1,2, Yong Cheng1, Jinyu Wang2*, Qiuling Peng3* \\ ${ }^{1}$ College of Veterinary Medicine, Yangzhou University/Jiangsu Co-innovation Center for Prevention and Control \\ of Important Animal Infectious Diseases and Zoonoses, Yangzhou, China \\ ${ }^{2}$ College of Animal Science and Technology, Yangzhou University, Yangzhou, China \\ ${ }^{3}$ Department of Biological and Chemical Engineering, Yichun University, Yichun, China \\ Email: "jywang@yzu.edu.cn, "978353865@qq.com
}

Received 11 June 2016; accepted 22 July 2016; published 26 July 2016

Copyright (C) 2016 by authors and OALib.

This work is licensed under the Creative Commons Attribution International License (CC BY).

http://creativecommons.org/licenses/by/4.0/

(c) (i) Open Access

\begin{abstract}
Targeted mutagenesis by zinc-finger nucleases (ZFNs) can be used to generate knock-out mammalian cell lines with high efficiency. A number of different methods have been developed for the design and assembly of gene-specific ZFNs, making them easily accessible to researchers. In this study, we used ZFNs assembled through the CoDA (context-dependent assembly) platforms to generate mutant caprine fetal fibroblasts cells for the BLG gene. ZFN plasmid was introduced into the caprine fetal fibroblasts cell by electroporation. ZFN-induced cleavage of the target sequence was confirmed by the Surveyor nuclease assay analysis. Sequence analysis revealed that ZFNinduced mutations such as base insertion, deletion, or substitution were generated in the ZFN cleavage site of BLG. The simplicity and efficacy of CoDA will enable broad application of ZFN technology. This technique could be used with homologous arm, which may target foreign genes into the BLG locus at higher efficiency.
\end{abstract}

\section{Keywords}

Context-Dependent Assembly, Zinc Finger Nucleases, Beta-Lactoglobulin, Gene Knockout, Goat

Subject Areas: Cell Biology, Genomics

\footnotetext{
${ }^{*}$ Corresponding authors.
}

How to cite this paper: Yuan, Y.G., Cheng, Y., Wang, J.Y. and Peng, Q.L. (2016) Targeted Mutagenesis of Beta-Lactoglobulin Gene in Caprine Fetal Fibroblasts by Context-Dependent Assembly Zinc-Finger Nucleases. Open Access Library Journal, 3: e2813. http://dx.doi.org/10.4236/oalib.1102813 


\section{Introduction}

Zinc finger nucleases (ZFNs) are artificial, hybrid restriction enzymes created by covalently linking a DNAbinding zinc finger (Zif) domain (composed of three to six finger-arrays) to the non-specific DNA cleavage domain (or simply FN) of the Flavobacterium okeanokoites bacterial restriction endonuclease-Fok I. ZFNs have recently become a powerful tool for genome modification since they are able to generate site-specific doublestrand breaks (DSBs) [1]. Repair of DSBs provides the molecular basis for gene disruption, gene correction, or gene addition during ZFN-mediated genome editing [2]. Specifically, a given DSB may be repaired by nonhomologous end joining (NHEJ) or homology-directed repair (HDR) pathways [1]. HDR utilizes a homologous donor sequence as template for the conservative repair of the DNA break. Provision of a suitably designed donor DNA molecule can, therefore, specify gene addition or gene correction at the ZFN-induced DSB [3]-[5]. NHEJ, on the other hand, catalyzes the rejoining of the two DNA ends, a process that can result in the deletion or insertion of nucleotides at the repair junction. DNA repair via NHEJ is, therefore, mutagenic [6]-[9]. To date, ZFN-induced DNA repair via HDR or NHEJ has been utilized to target modifications to the genomes of numerous species [10].

There are some options for designing and assembling ZFN for specific genes. Ready to use expression plasmids can be purchased from commercial sources (e.g. ZFNs from Sigma-Aldrich) or assembled in the laboratory using publicly available resources, such as OPEN, CoDA, and modular-assembly for ZFNs. Selection assays for identifying zinc fingers that bind specific targets are laborious and challenging for non-specialist laboratories [10]. An alternative method, known as modular assembly, combines pre-selected zinc-finger modules into arrays. These ZFNs are relatively easy to generate but have low success rates [11], although significant progress has recently been made. Proprietary methods have also been used to generate ZFNs that are effective in organisms [5]-[9] [12], but these nucleases must be purchased and are expensive. While assembling ZFNs through OPEN and modular assembly are technically challenging and labor-intensive, the CoDA approach is straightforward and requires two straight-forward steps. The first is a computational analysis of the target gene to identify potential ZFP binding sites, and the second involves the synthesis and cloning of the ZFP coding sequences into appropriate expression vectors containing the heterodimeric Fok1 nuclease domain. These procedures can be completed in 1 to 2 weeks without the need for labor-intensive selection which can be moved directly into cells for testing as ZFNs. Therefore, in our study, we used the CoDA approach for designing ZFNs. $\beta$-lactoglobulin (BLG) is a major ruminant milk protein and is an ideal locus in mammary gland bioreactors for producing complex bioactive proteins. Yu et al. [13] had successfully applicated ZFNs to knockout BLG in cattle with high effciency. In this study, we describe methods to analyze caprine BLG gene mutations in cultured cells introduced by expression of ZFNs.

\section{Materials and Methods}

\subsection{Chemicals}

All chemicals were purchased from Sigma-Aldrich Chemical (St. Louis, MO, USA), unless otherwise indicated.

\subsection{Design and Assembly of CoDA ZFNs}

ZFN plasmids were designed to bind and cleave intron 3 of the caprine $\beta$-lactoglobulin gene (Z33881.1), using the ZiFIT CoDA Targeter (http://zifit.partners.org/ZiFiT) [8] [14] [15]. The full binding site of the ZFN pair used is 4761-ACCCCCCTTCCCTTGCTGGGCAGTGT-4786, where the uppercase letters are the ZFN binding sites and the lowercase letters are where the double-strand break was created. DNA fragments encoding zincfinger arrays (Table 1) were synthesized (shanghaishenggong Inc, Shanghai, China) and cloned into FokI EL/KK heterodimeric expression vectors by BamHI/XbaI (pMLM290 and pMLM292). Each ZFN contained three zinc fingers. Plasmids were obtained from the non-profit plasmid repository Addgene.

\subsection{Preparation of Cells and Culture Conditions}

Fetal fibroblasts were obtained from 35-day-old fetuses that were produced by mating nanjiang yellow goats. The head, viscera and bones of each fetus were removed and washed three times using Dulbecco's phosphate buffered saline (D-PBS). The washed tissue was finely chopped into fragments smaller than $1 \mathrm{~mm}$ in size, and 
Table 1. Zinc fingers used in ZFN (CoDA source)

\begin{tabular}{cccc}
\hline Finger & Helix & Triplet & $\begin{array}{c}\text { Reference } \\
\text { Number }\end{array}$ \\
\hline Left F1 & RKHHLGR & GGG & C12-211 \\
Left F2 & RREHLVR & GGG & C12-211 \\
Left F3 & QDGNLGR & GAA & C23-212 \\
Right F1 & RSHILTN & GTG & C12-103 \\
Right F2 & QSTTLKR & GCA & C12-103 \\
Right F3 & RSDHLSL & TGG & C23-106 \\
\hline
\end{tabular}

the tissue fragments were placed in 35-mm culture dishes (Falcon, BD, USA) and incubated for 30 min in a humidified incubator at $38.5^{\circ} \mathrm{C}$ with $5 \% \mathrm{CO}_{2}$. After confirming that the tissue fragments adhered to the culture dish, a high-glucose Dulbecco's modified Eagle medium (HyClone, Beijing, China) containing 10\% FCS (HyClone, Beijing, China) was gently added. After 2 - 3 days, the tissue fragments were removed, and the cells adhering to the bottom of the culture dish were cultured. After two to three passages, the cells were stored frozen based on conventional methods.

\subsection{ZFNs Plasmid Transfection}

For ZFNs transfection, the cells were seeded at $3 \times 10^{5}$ cells per well in 24-well dishes. The next day, cells were transfected with $15 \mu \mathrm{g}$ of each ZFN expression plasmid (in pairs) by electrotransfection. For the mutation analysis, 24 hours post-transfection, cells were divided into two groups. One group was the mixed cells $\left(10^{6}\right)$ and the total DNA was extracted for PCR and sequenced. The other group $\left(10^{4}\right)$ was to form single colonies by limited dilution ( 1 cell per well on average). The fetal fibroblasts were cultured to $70 \%-90 \%$ confluency (after $10-14$ d culture), then washed twice with D-PBS (-) and treated with $0.05 \%$ trypsin-EDTA to isolate and collect the cells. In this study, ZFNs plasmid transfer experiment was repeated at least three times.

\subsection{Surveyor Nuclease Assay (Mutation Detection Assay)}

ZFN-induced mutations were detected using the SURVEYOR Mutation Detection Kit (Transgenomic, Omaha, USA) based on the manufacturer's protocol. The assay detects non-homologous end-joining (NHEJ)-mediated imperfect repair of ZFN-induced double-strand breaks (DSBs) by digesting hetero duplexes consisting of wild-type and mutant DNA with Surveyor nuclease, a mismatch DNA-specific cleavage enzyme [13]. Briefly, $24 \mathrm{~h}$ after electroporation, genomic DNA was extracted from the ZFN-treated and control cells using a DNA extraction kit (Shanghai Ruifeng Agro-tech, Shanghai, China). The genomic DNA was used as a template, and a region recognized and cleaved by ZFNs was amplified by PCR. The PCR was performed using PrimeSTAR HS DNA polymerase (Takara, Dalian, China), a high-fidelity enzyme, under the following conditions: $95^{\circ} \mathrm{C}$ for 1 min, followed by $95^{\circ} \mathrm{C}$ for $30 \mathrm{~s}, 65^{\circ} \mathrm{C}$ for $30 \mathrm{~s}$, and $72^{\circ} \mathrm{C}$ for 1 min for 30 cycles. The sequences of the PCR primers used are as follows: 5'-TCGTCCTAAATCCG AGATGAGAAAG-3' and 5'-CTTCCTCTTCCCTGCTGC CACTGCC-3'. The cell-derived and the control cell-derived amplicons were obtained by PCR and, along with a mixture of both, were heat denatured, allowed to rehybridize, and then digested by the Surveyor nuclease. The digested samples were subjected to polyacrylamide gel electrophoresis to confirm ZFN-induced mutations. This assay was performed in all three ZFNs transfection experiments.

\subsection{Analysis of ZFN-Induced Mutations by DNA Sequencing}

Genomic DNA was extracted from single clone cells (cloned from ZFN-treated cells) and mixed cells, and used as a template for PCR to amplify the region recognized and cleaved by the ZFNs. The amplification products from mixed cells were cloned into pGEM-T Easy Vector (Promega, Madison, USA). Plasmids were extracted from the resultant $E$. coli colonies for DNA sequencing. 


\section{Results and Discussion}

Traditionally, creating a gene knockout animal is solely dependent on the availability of embryonic stem cell lines that have only been established in mouse and rat [1]. The low efficiency of gene targeting in cultured somatic cells is the main barrier for gene targeting in large animals. Recently, zinc-finger nuclease (ZFN) technology has provided powerful tools for editing genomes of any animals [10]. The success of ZFN technology for gene targeting in many organisms (e.g. insects, amphibians, plants, nematodes), and several mammals (e.g. humans) encouraged us to establish a high-efficiency gene-targeting platform in large animals such as goats. The CoDA method for designing a pair of ZFNs in this report provide a rapid, highly effective, and publicly available platform for engineering zinc-finger arrays [7] [14]-[16]. To mutate the BLG gene (Z33881.1) in goat, we designed one pair of ZFNs that targets the caprine BLG gene using the CoDA method. When the coding sequence of BLG was analyzed by the ZiFIT CoDA Targeter with parameter of 5 - 6 spacers, the targeted sequence of all ZFNs were located in the introns. Since our later aim was to obtained transgenic cell, which foreign gene were knocked in at BLG locus by homologous recombination co-transfection with ZFNs, the ZFNs for intron 3 but not for exon were choosed and used for gene knocked-in. To test the activity of the pair of ZFNs, we evaluated each for its ability to bind to its cognate DNA target site using a well-established bacterial two-hybrid (B2H) reporter assay (data not show). The two ZFAs for the intron 3 target sites functioned effectively as ZFNs and were further subjected for testing in caprine fetal fibroblast cells. Fetal fibroblasts were transfected with the ZFN plasmid. A surveyor nuclease assay was performed on mixed cells the day after electroporation. It was expected, depending on the type of mutation induced by ZFN treatment, that if mutations had occurred in the BLG target sequence, fragments of approximately $378 \mathrm{bp}$ and $157 \mathrm{bp}$ would be produced by digestion using the Surveyor nuclease. In agreement with this expectation, two bands of approximately 378 bp and 157 bp were detected in the ZFN-treated cell-derived amplicon (Figure 1). By contrast, no bands were detected near 535 bp in the amplicon from control cells. These results show that mismatch DNA was present in the ZFN-treated cell-derived amplicon, confirming the presence of mutations induced by ZFN treatment. This experiment confirmed that ZFNs with CoDA method can induce cleavage and mutation in the target sequences. CEL I assay revealed 3.14\% ZFN activity (Figure 1), indicating 3.14\% mutated alleles in the pooled cell population. Similar results were obtained in all of the three plasmid transfer experiments.

Functional genomics, the use of gene knock-out or knock-in techniques, is severely limited by methods for rapid targeting and disrupting of a gene of interest. Early approaches to somatic cell gene disruption used genome-wide non-targeted methods, including ionizing radiation and chemical-induced mutagenesis, whereas more recent methods used targeted homologous recombination (HR). However, the $>1000$-fold lower frequency of the targeted HR event relative to random integration in most mammalian cell lines (beyond mouse ES cells) can necessitate screening thousands of clones and take several months to identify a biallelic targeted gene knockout. Strategies including positive and negative marker selection and promoter-trap can considerably boost efficiencies, although these approaches present their own technical challenges and are not always successful in achieving high efficiency targeting [3]. Although advances with adeno-associated viral delivery strategies continue to improve knockout efficiency, the frequency is still very low and the time required to achieve biallelic

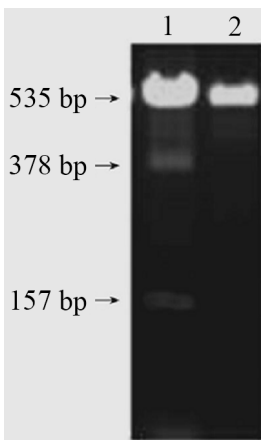

Figure 1. Confirmation of ZFN-induced mutations by the Surveyor nuclease assay. 1. Amplicons from ZFN-treated cells, a 535-bp PCR product was cleaved into two fragments: 378 bp and 157 bp; 2. Control cells. 
gene knockout remains a barrier to its routine adoption [10].

The use of ZFN can greatly facilitate the mutation or integration of a transgene at a precise genomic locus [2]. In order to select mutated cells, 264 isolates cloning were analyzed via direct sequencing of PCR products of the genomic target locus. The presence of double peaks after the targeting site in the sequencing curves clearly distinguishes mutants from non-targeted cells (Figure 2). Various mutations were identified in nine of the 264 clones (98 from fibroblast cells and 166 from E. coli clones; 3.4\% efficiency) (Figure 3), as follows: 5 bp deletion in clones 1-A4 and 1-C4, 2 bp substitution in clones 1-H5 and 4-C6, 1 bp substitution +2 bp deletion (clone 7-E2), 2 bp deletion (clone 3-F10), and 3 bp insertion (clone 3-C9). Two were found to be homozygous mutations, with the remaining seven clones being heterozygous. All mutations were centered on the ZFN cleavage site, and were consistent with intron 3 . The types of mutation recovered were consistent with those already reported, such as deletions, substitutions and insertions [8] [9] [13] [17]-[20]. To test whether ZFN plasmid randomly integrated into the goat genome, PCR was performed to examine ZFN plasmid integration by amplifying CMV-FokI catalytic domain from all mutant clones. The desired sequence was not found in nine clones that were screened (data not shown). For further proof-of-concept that the introduction of foreign genes in the BLG locus with ZFNs for nuclear donor cells to produce transgenic goats, different lengths of homologous arms would be prepared for cotransfection.

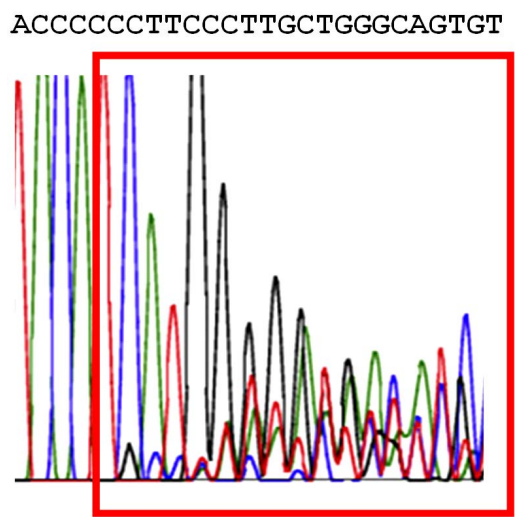

Figure 2. DNA sequencing revealed the mutation at ZFN targeted region. To detect mutations at $\mathrm{ZFN}$ targeted region in a single-cell colony, the PCR products of a single-cell colony were directly sequenced. If the colony is a mutant clone, the peak after the ZFNs cutting site should be double peaks (red) box).

Insertion

Clone:

3-C9 4761-ACCCCCCTTCCCACGTTGCTGGGCAGTGT-4786

WT

4761-ACCCCCCTTCCC . . TTGCTGGGCAGTGT-4 786

Deletions, substitutions, complex:

$\begin{array}{ll}\text { 1-A4, 1-C4 } & 4761-\text { ACCCCC-----CTTGCTGGGCAGTGT-4786 } \\ \text { 3-F10 } & 4761-\text { ACCCCCCTTCCCTTGC--GGCAGTGT-4786 } \\ \text { 7-E2 } & 4761-\text { ACC--CCTTTCCTTGCTGGGCAGTGT-4786 } \\ \text { 1-H5 } & 4761-\text { ACCCCCCTTCCAGTGCTGGGCAGTGT-4786 } \\ \text { 4-C6 } & 4761-\text { ACCCCCCTTCCCTTATTGGGCAGTGT-4786 } \\ \text { WT } & 4761 \text {-ACCCCCCTTCCCTTGCTGGGCAGTGT-4786 }\end{array}$

Figure 3. ZFN-induced mutations in clones of cells. WT indicates the wild-type (non-mutated) Sequence; Substituted bases and inserted bases are indicated by black; Deleted bases are indicated by “-”. 


\section{Conclusion}

This study demonstrates that CoDA provides an effective alternative method for using publicly available reagents to engineer ZFNs. Therefore, this strategy could be designed against an endogenous genomic locus with high efficiency. Creation of goats with targeted gene disruption or knock-in has been hampered by the inefficiencies of relying on homologous recombination to create a single disrupted allele. The results presented here suggest that ZFNs are promising approach for specific gene editing in goat and can be used in combination with an appropriately structured donor DNA for targeted transgene integration into the BLG locus for expression of foreign proteins.

\section{Acknowledgements}

This study was supported by A Project Funded by the Priority Academic Program Development of Jiangsu Higher Education Institutions (PAPD), China Postdoctoral Science Foundation (2015M571828), Jiangsu Postdoctoral Science Foundation (1402001C) and 2014 domestic production, collaborative innovation (2014-7).

\section{Approved}

Our study is approved by ethics committee of Yangzhou university.

\section{Conflict}

On behalf of all authors, the corresponding author states that there is no conflict of interest.

\section{References}

[1] Provost, F.L., Lillico, S., Passet, B., Young, R., Whitelaw, B. and Vilotte, J.L. (2009) Zinc Finger Nuclease Technology Heralds a New Era in Mammalian Transgenesis. Trends Biotechnology, 28, 134-141. http://dx.doi.org/10.1016/j.tibtech.2009.11.007

[2] Urnov, F.D., Rebar, E.J., Holmes, M.C., Zhang, H.S. and Gregory, P.D. (2010) Genome Editing with Engineered Zinc Finger Nucleases. Nat Reviews Genetics, 11, 636-646. http://dx.doi.org/10.1038/nrg2842

[3] Rémy, S., Tesson, L.S., Ménoret, C., Usal, A., Scharenberg, M. and Anegon, S. (2010) Zinc-Finger Nucleases: A Powerful Tool for Genetic Engineering of Animals. Transgenic Research, 19, 363-371. http://dx.doi.org/10.1007/s11248-009-9323-7

[4] Lei, Y., Lee, C. L., Joo, K., Zarzar, J., Liu, Y., Dai, B., Fox, V. and Wang, P. (2011) Gene Editing of Human Embryonic Stem Cells via an Engineered Baculoviral Vector Carrying Zinc-Finger Nucleases. Molecular therapy, 19, 942-950. http://dx.doi.org/10.1038/mt.2011.12

[5] Meyera, M., Angelis, M.H., Wursta, W. and Kühn, R. (2010) Gene Targeting by Homologous Recombination in Mouse Zygotes Mediated by Zinc-Finger Nucleases. Proceedings of the National Academy of Sciences of the USA, 107, 15022-15026. http://dx.doi.org/10.1073/pnas.1009424107

[6] Lutz, A.J., Li, P., Estrada, J.L., Sidner, R.A., Chihara, R.K., Downey, S.M., Burlak, C., Wang, Z.Y., Reyes, L.M, Ivary, B., Yin, F., Blankenship, R.L., Paris, L.L. and Tecto, A.J. (2013) Double Knockout Pigs Deficient in N-Glycolylneuraminic Acid and GALACTOSE a-1, 3-Galactose Reduce the Humoral Barrier to Xenotransplantation. Xenotransplantation, 20, 27-35. http://dx.doi.org/10.1111/xen.12019

[7] Yang, D., Yang, H., Li, W., Zhao, B., Ouyang, Z., Liu, Z., Zhao, Y., Fan, N., Song, J., Tian, J., Li, F., Zhang, J., Chang, L., Pei, D., Chen, Y.E. and Lai, L. (2011) Generation of PPAR Gamma Mono-Allelic Knockout Pigs via Zinc-Finger Nucleases and Nuclear Transfer Cloning. Cell Research, 21, 979-982. http://dx.doi.org/10.1038/cr.2011.70

[8] Whyte, J.J. and Prather, R.S. (2011) Zinc Finger Nucleases to Create Custom-Designed Modifications in the Swine (Sus scrofa) Genome. Journal Animal Science, 90, 1111-1117. http://dx.doi.org/10.2527/jas.2011-4546

[9] Hauschilda, J., Petersena, B., Santiagob, Y., Queissera, A.L., Carnwatha, J.W., Lucas-Hahna, A., Zhang, L., Meng, X., Gregory, P.D., Schwinzerd, R., Costb, G.J. and Niemanna, H. (2011) Efficient Generation of a Biallelic Knockout in Pigs Using Zinc-Finger Nucleases. Proceedings of the National Academy of Sciences of the USA, 108, 2013-2017. http://dx.doi.org/10.1073/pnas.1106422108

[10] Hauschild-Quintern, J., Petersen, B., Cost, G.J. and Niemann. H. (2012) Gene Knockout and Knockin by Zinc-Finger Nucleases: Current Status and Perspectives. Cellular Molecular Life Sciences, 70, 2969-2983.

http://dx.doi.org/10.1007/s00018-012-1204-1 
[11] Ramirez, C.L., Foley, J.E., Wright, D.A., Muller-Lerch, F., Rahman, S.H., Cornu, T.I., Winfrey, R.J., Sander, J.D., Fu, F., Townsend, J. A., Cathomen, T., Voytas, D.F. and Joung, J.K. (2008) Unexpected Failure Rates for Modular Assembly of Engineered Zinc-Fingers. Nature Methods, 5, 374-375. http://dx.doi.org/10.1038/nmeth0508-374

[12] Whyte, J.J., Zhao, J., Wells, K.D., Samuel, M.S., Whitworth, K.M., Walters, E.M., Laughlin, M.H. and Prathe, R.S. (2011) Gene Targeting with Zinc Finger Nucleases to Produce Cloned eGFP Knockout. Molecular Reproduction Development, 78, 2. http://dx.doi.org/10.1002/mrd.21271

[13] Yu, S., Luo, J., Song, Z., Ding, F., Dai, Y. and Li, N. (2011) Highly Efficient Modification of Beta-Lactoglobulin (BLG) Gene via Zinc-Finger Nucleases in Cattle. Cell Research, 21, 1638-1640. http://dx.doi.org/10.1038/cr.2011.153

[14] Chen, S., Oikonomou, G., Chiu, C.N., Niles, B.J., Liu, J., Lee, D.A., Antoshechkin, I. and Prober, D.A. (2013) A large-Scale in Vivo Analysis Reveals That TALENs Are Significantly More Mutagenic than ZFNs Generated Using Context-Dependent Assembly. Nucleic Acids Research, 41, 2769-2778. http://dx.doi.org/10.1093/nar/gks1356

[15] Sood, R., Carrington, B., Bishop, K., Jones, M., Rissone, A., Candotti, F., Chandrasekharappa, S.C. and Liu, P. (2013) Efficient Methods for Targeted Mutagenesis in Zebrafish Using Zinc-Finger Nucleases: Data from Targeting of Nine Genes Using CompoZr or CoDA ZFNs. PloS One, 8, e57239. http://dx.doi.org/10.1371/journal.pone.0057239

[16] Sander, J.D., Dahlborg, E.J., Goodwin, M.J., Cade, L., Zhang, F., Cifuentes, D., Curtin, S.J., Blackburn, J.S., Thibodeau-Beganny, S., Qi, Y., Pierick, C. J., Hoffman, E., Maeder, M.L., Khayter, C., Reyon, D., Dobbs, D., Langenau, D.M., Stupar, R.M., Giraldez, A.J., Voytas, D.F., Peterson, R.T., Yeh, J.R.J. and Joung, J.K. (2011) Selection-Free Zinc-Finger-Nuclease Engineering by Context-Dependent Assembly (CoDA). Nature Methods, 8, 67-69. http://dx.doi.org/10.1038/nmeth.1542

[17] Kwon, D. N., Lee, K., Kang, M. J., Choi, Y.J., Park, C., Jeffrey, J., Brown, A.N., Kim, J.H., Samue, M., Mao, J., Park, K.W., Murphy, C.N., Prather, R.S. and Kim, J.H. (2013) Production of Biallelic CMP-Neu5Ac Hydroxylase Knock-Out Pigs. Scientific Reports, 3, 1981-1990. http://dx.doi.org/10.1038/srep01981

[18] Li, P., Estrada, J.L., Burlak, C. and Tector, A.J. (2013) Biallelic Knockout of the Alpha-1, 3 Galactosyltransferase Gene in Porcine Liver-Derived Cells Using Zinc Finger Nucleases. Journal of Surgical Research, 181, 39-45. http://dx.doi.org/10.1016/j.jss.2012.06.035

[19] Toscano, M.G., Anderson, P., Muñoz, P., Lucena, G., Cobo, M., Benabdellah, K., Gregory, P.H., Holmes, M.C. and Martin, F. (2013) Use of Zinc-Finger Nucleases to Knock out the WAS gene in K562 Cells: A Human Cellular Model for Wiskott-Aldrich Syndrome. Disease Models Mechanisms, 6, 544-554. http://dx.doi.org/10.1242/dmm.010652

[20] Cao, S.Z., Yue, C.H, Li, X.R., Feng, C., Long, C. and Pan, D. K. (2013) Production of Myostatin Gene Knockout Wuzhishan Miniature Pig Fibroblasts with Zinc-Finger Nucleases. HEREDITAS, 35, 778-785. http://dx.doi.org/10.3724/SP.J.1005.2013.00778

\section{Submit or recommend next manuscript to OALib Journal and we will provide best service for you:}

- Publication frequency: Monthly

- 9 subject areas of science, technology and medicine

- Fair and rigorous peer-review system

- Fast publication process

- Article promotion in various social networking sites (LinkedIn, Facebook, Twitter, etc.)

- Maximum dissemination of your research work

Submit Your Paper Online: Click Here to Submit

Contact Us: service@oalib.com 